DR. HENNING HOLLE (Orcid ID : 0000-0003-2455-1053)

Article type : Regular Article

\title{
Transcranial magnetic stimulation over contralateral primary somatosensory cortex disrupts perception of itch intensity
}

Olivia Jones, Igor Schindler, \& Henning Holle

Department of Psychology, University of Hull, U.K.

Corresponding author: Dr Henning Holle, Department of Psychology, Faculty of Health Sciences, University of Hull, Cottingham Road, Hull, HU6 7RX, U.K., Telephone: +44 (0)1482 466152, e-mail: h.holle@hull.ac.uk

Brief running title: TMS over S1 disrupts perception of itch intensity

Conflict of Interest: None declared.

Submitted to Experimental Dermatology, for consideration of the focus theme "Pruritus and prurigo dermatoses" (Guest Editors: S. Ständer and G. Yosipovitch)

\section{Abstract}

Itch, a complex unpleasant sensation causing the desire to scratch, results from the activity of a network of brain regions. However, the specific functional contributions of individual regions within this network remains poorly understood. We investigated whether contralateral primary and secondary somatosensory cortices (S1, S2) and ipsilateral inferior frontal gyrus (IFG) are critically involved in the cortical processing of acute itch. Continuous theta-burst transcranial magnetic stimulation (cTBS) was applied to either S1, S2 or IFG, followed by itch induction using a histamine prick. Results indicate a significant reduction of itch intensity when cTBS was applied to S1. Stimulation of S2 or the IFG were not associated with a significant reduction in itch intensity. The novel finding of an anti-pruritic effect elicited by disruption of activity in contralateral S1 suggests a causal role of $\mathrm{S} 1$ in encoding the sensory-discriminative aspect of itch and might be important in

This article has been accepted for publication and undergone full peer review but has not been through the copyediting, typesetting, pagination and proofreading process, which may lead to differences between this version and the Version of Record. Please cite this article as doi: 10.1111/exd.13803

This article is protected by copyright. All rights reserved. 
future studies on brain interventions for the treatment of itch.

Key words: antipruritic; histamine; pruritus; TMS, somatosensory; meta-analysis; cerebral itch response

\section{Introduction}

Itch is a complex sensory experience resulting from activity of a network of brain regions. However, the functional contribution of individual regions in this network remains poorly understood. Contralateral somatosensory cortices ( $\mathrm{S} 1$ and $\mathrm{S2}$ ), bilateral supplementary motor area (SMA), insula and anterior cingulate cortices (ACC) as well as ipsilateral inferior frontal gyrus (IFG) are consistently activated across studies (for review, see ${ }^{[1]}$, as well as the quantitative meta-analysis performed within the present study). It has been argued that functional specialization exists within this cortical network, with a sensory-discriminative role for somatosensory cortices, an affective-motivational role for insula and ACC, and a role of motor planning of the associated scratch response for SMA and IFG ${ }^{[2]}$. However, such functional specialization has been demonstrated only for the related domain of pain, whereas evidence for causal links between brain areas and specific functions in itch is currently sparse. One method ideally suited to identify brain areas causally involved in a given process is transcranial magnetic stimulation (TMS). If a brain area is critically involved in the sensory aspect of itch, then perturbing its activity using TMS should reduce itch intensity.

To date, only two brain stimulation studies on itch have been conducted. Both of these studies used transcranial direct current stimulation (tDCS) which delivers a low intensity current to a brain area via electrodes on the scalp. In tDCS, cathodal stimulation is assumed to decrease cortical excitability, whereas anodal stimulation increases excitability. Knotkova et al. ${ }^{[3]}$ reported a single-case study of a patient with syringomelia, accompanied by treatment-resistant chronic itch and pain. Repeated cathodal stimulation of the somatosensory cortex reduced itch, but not pain, for several months suggesting that repeated tDCS stimulation may counteract cortical hyperexcitability associated with chronic itch. A second study by Nakagawa et al. ${ }^{[4]}$ investigated the effects of tDCS stimulation of S1 on histamine-induced acute itch in healthy volunteers. In the two experimental conditions, the cathode was either placed over ipsilateral S1 and the anode over contralateral S1, or vice versa. Compared to sham stimulation, both experimental conditions temporarily reduced itch intensity. However, due to the bi-hemispheric nature of their stimulation, the relative contribution of ipsi- and contralateral S1 on top-down itch inhibition could not be ascertained. Another limitation is that due to the size of the electrodes employed $(5 \times 5 \mathrm{~cm})$, stimulation was not restricted to the intended region, but inevitably also involved neighbouring cortical areas (e.g., motor cortex).

Thus, a primary aim of the present study is to investigate whether a focal, temporary disruption of activity in contralateral S1 using continuous theta-burst TMS $\left(\right.$ cTBS, $\left.{ }^{[5]}\right)$ leads to a reduction of acute itch intensity. Given that a sensory-discriminative role for $\mathrm{S} 2$ in itch has been suggested, and

This article is protected by copyright. All rights reserved. 
evidence that TMS of the contralateral S2 reduces acute pain ${ }^{[6,7]}$, this area was also stimulated in our study. Finally, we were interested in whether CTBS of IFG, an area suggested to be involved in sustained attention, the cognitive dimension of itch and/or the planning of a scratching response ${ }^{[8-}$ ${ }^{10]}$ would lead to a reduction of itch intensity. Answering these questions will not only inform current theories about critical components of the cortical itch network, but is also necessary for future brain-based itch interventions.

\section{Materials and Methods}

\section{Participants}

A total of 19 participants ( 6 male, mean age $25.5 \pm 5.7)$ took part in the study. All were right handed as determined by the Edinburgh Handedness Questionnaire (Mean Laterality

Coefficient $=82.13, \mathrm{SD}=20.62,{ }^{[11]}$ ), had normal or corrected-to-normal vision and had no history of neurological or psychiatric disorders. Participants were free from any sensitivity on the right volar forearm (e.g. wounds, rashes, swelling or reddening), were free from skin conditions (e.g. eczema), disease of the heart or blood vessels (e.g. cardiovascular disease), and lastly, had not taken any antihistamines 48 hours prior to the experimental session. The study was approved by the University of Hull Psychology Ethics Committee and performed according to the British Psychology Society's code of human research ethics principles. All participants took part in a trial TMS session prior to giving written informed consent to take part in the study. Participation was remunerated at a rate of $£ 8$ per hour. All participants were fully debriefed at the end of the last experimental session.

\section{Experimental design}

The experiment consisted of four one hour TMS sessions, approximately one week apart. In each session, a brain region was stimulated for 40 seconds, followed by a histamine prick test and 10 minutes of itch intensity ratings (see below). Order of the four TMS sessions was counterbalanced across participants, using a Latin Square design balanced for first order carryover effects (see http://statpages.info/latinsq.html $)^{[12]}$.

This article is protected by copyright. All rights reserved. 


\section{TMS procedure}

The regions targeted for TMS were determined based on a quantitative meta-analysis of neuroimaging studies on acute itch. This meta-analysis (see supplementary online material) indicated that left S1 (MNI coordinates: $-32,-35,64)$, left S2 $(-47,-21,13)$ and the right IFG $(51,10,7)$ are consistently activated across studies when participants are experiencing itch on their right volar forearm. Bilateral insular cortices and cingulate cortex were also identified in this meta-analysis, however, such more deeply located brain regions cannot be stimulated in a focal manner due to the depth-focality trade-off in TMS ${ }^{[13]}$. We chose to not use vertex stimulation as control condition, since the meta-analysis indicated that the supplementary motor area (SMA) is also consistently activated during acute itch and vertex stimulation may inadvertently disrupt SMA activity. Instead, we chose the hemispheric gap over the Superior Parietal Lobe (SPL, -3, -62, 71) as a control site, since this brain region was never activated in any of the reviewed neuroimaging studies.

To enable an image-guided TMS navigation, high resolution $(1 \times 1 \times 0.6 \mathrm{~mm})$ T1 weighted anatomical MRI scans of each participant were acquired at Hull Royal Infirmary using a GE medical systems scanner with a field strength of 3 Tesla. MNI coordinates of the 3 target areas and the control area were defined as regions of interest (ROIs) using Marsbar (marsbar.sourceforge.net) and SPM12 (see Fig. 1). These ROIs were then backprojected from MNI space into each participant's native brain space, using SPM12's inverse transformation function. Subject-specific ROIs were then imported into BrainVoyager and superimposed on the surface reconstruction of the two hemispheres and defined as targets during neuronavigation, using the Brain Voyager TMS Neuronavigator system (version 2.2.0). This ensured precise stimulation of each target region in each participant.

A Magstim Rapid ${ }^{2}$ stimulator was used to generate repetitive magnetic pulses. The pulses were delivered with a standard $70 \mathrm{~mm}$ figure-8 coil. For both IFG and S2 stimulation the coil was positioned firmly against the temple, centered over right IFG or left S2 and oriented such as to maximise current flow in the anterolateral direction, i.e. perpendicular to the right precentral sulcus (for IFG) or left postcentral sulcus (for S2). For stimulation of left S1, the coil was placed tangentially on the skull with the coil orientation for S1 guided by the angle of the left postcentral gyrus. SPL was stimulated using a coil orientation of 0 degrees, i.e. perpendicular to the longitudinal cerebral fissure. A continuous theta burst rTMS train of 804 pulses was used ( 268 bursts, each burst consisting of three pulses at $30 \mathrm{~Hz}$, repeated at intervals of $100 \mathrm{~ms})^{[14,15]}$. Stimulation intensity was set to $40 \%$ of maximum machine output, corresponding on average to $104 \%$ of active motor threshold ${ }^{[16]}$, and lasted for $40 \mathrm{~s}$. Directly after theta burst stimulation, the histamine prick test was conducted, followed by 10 minutes of itch intensity ratings.

\section{Itch induction and rating procedure}

The histamine prick test consisted of the application of a single drop of $1 \%$ histamine dihydrochloride to the right volar forearm ${ }^{[8,17]}$. The skin was then pricked through this drop with the tip of a sterile lancet (ALK, Sweden). A visual barrier was used so that participants could not see their hand during itch induction and rating, in order to control a potential confounding variable (i.e. looking at one's own reddened or swelling skin might be itch-inducing in itself', see ${ }^{[18]}$ ). Starting with the onset of the histamine prick, participants rated itch intensity every 15 seconds for a duration of 10 minutes

This article is protected by copyright. All rights reserved. 
using the general labelled magnitude scale ${ }^{[g L M S},{ }^{19]}$ which provides more reliable estimates of acute itch intensity ${ }^{[20]}$. The gLMS ranges from $0-100$, with verbal anchors 'no itch' at 0 , 'barely detectable' at 1 , 'weak' at 6 , 'moderate' at 17 , 'strong' at 35, 'very strong' at 53 and 'strongest itch imaginable' at 100 . Only the verbal anchors were visible to participants, not the numbers. The rating scale was presented on a computer running Presentation (version 17.2, www.neurobs.com). Participants were instructed to use the whole scale, not just the labelled points. Prior to taking part in the TMS sessions, each participant took part in a familiarization session, where they learnt the correct use of the scale, as recommended by ${ }^{[21]}$, and could experience the potential novel sensation of histamine-induced itch.

\section{Data analysis}

Ratings were normalized by dividing each obtained rating by the overall peak rating given by that participant across all four experimental sessions. This transformation takes into account the different use of the rating scale by the participants ${ }^{[22]}$ which can be a considerable source of variation in rating scales ${ }^{[23]}$. The obtained normalized itch scores range between 0 and 1 , and can be interpreted as the percentage of maximum itch. For the statistical analysis, we calculated the mean across each entire normalized time series (i.e., using all 40 ratings), separately for each session of each participant. We also extracted the peak of each time series as an additional outcome variable. Since we had a-priori hypotheses for the experiment, focussing on the comparison of each stimulation condition with control site stimulation, we favoured a more sensitive hypothesis-driven approach instead of an unspecific omnibus one-way ANOVA for the statistical analysis, as recommend by Tabachnick and Fidell ${ }^{[24]}$. Thus, to answer the research question of whether stimulation of either S1, S2 or the IFG significantly reduces itch intensity, relative to control site stimulation (SPL), the following three apriori planned contrasts were computed: SPL vs. S1, SPL vs. S2 and SPL vs. IFG. These contrasts were realized as two-tailed paired samples t-tests, corrected for multiple comparisons using the Bonferroni procedure.

\section{Results}

Itch ratings

As can be seen in Fig. 2, itch intensity peaked about $120 \mathrm{~s}$ after the histamine prick, followed by a slow decay. Relative to control site stimulation, itch intensity tended to be reduced for IFG, S1 and S2 stimulation, at least descriptively, with the effect being most pronounced when theta-burst TMS was applied to $\mathrm{S} 1$.

When using the peak of each time series as a summary statistic, it can be seen that relative to control site stimulation ( $\mathrm{M}=0.64,95 \% \mathrm{Cl}[0.45,0.83])$, itch intensity was reduced when $\mathrm{S} 1$ was stimulated ( $\mathrm{M}=0.35,95 \% \mathrm{Cl}[0.18,0.52])$. The planned contrast for this comparison was not significant after Bonferroni correction was applied $(t(18)=2.19, p=.126)$. The peaks for conditions S2 $(\mathrm{M}=0.46,95 \% \mathrm{Cl}[0.27,0.65])$ and IFG $(\mathrm{M}=0.45,95 \% \mathrm{Cl}[0.24,0.65])$ did not differ significantly from the control condition SPL (both $t<1.31$, both $p>.63$ ).

This article is protected by copyright. All rights reserved. 
A similar pattern emerges when using the mean of each time series. Relative to control site stimulation ( $\mathrm{M}=0.19,95 \% \mathrm{Cl}[0.11,0.26])$, mean itch intensity was reduced following S1 stimulation $(\mathrm{M}=0.10,95 \% \mathrm{Cl}[0.04,0.16])$. The corresponding planned contrast was statistically significant $(t(18)=2.64, p=.049)$ and is equivalent to a $47 \%$ reduction in mean itch intensity. The means for conditions S2 ( $\mathrm{M}=0.14,95 \% \mathrm{Cl}[0.07,0.21])$ and IFG $(\mathrm{M}=0.16,95 \% \mathrm{Cl}[0.07,0.24])$ did not differ significantly from the control condition (both $t<1.09$, both $p>.87$ ).

\section{Discussion}

The aim of the present study was to identify brain regions critically involved in the cortical processing of itch. The main finding is that relative to control site stimulation, continuous thetaburst TMS (cTBS) of S1 results in a significant reduction of itch intensity following itch induction via a histamine prick. cTBS stimulation of S2 or the IFG were not associated with a significant reduction in itch intensity.

While several neuroimaging studies have reported S1 activation when healthy volunteers experience an acute itch stimulus ${ }^{[8,10,17,25-27]}$, these studies, due to their correlational methodological nature, cannot unambiguously ascribe a specific function to a given activation. This is especially a challenge for itch research, since the sensation consists of multiple correlated (e.g., sensory, affective) dimensions and triggers involuntary planning and/or execution of itch-associated motor responses (e.g., scratching). The present study extends existing knowledge by demonstrating that in humans activation in contralateral S1 is critical, and not merely epiphenomenal, for the sensorydiscriminative aspect of acute itch.

By applying cTBS to S1, the excitability of neurons in this area is temporarily reduced ${ }^{[5]}$. Brodmann Area $3 \mathrm{~b}$, which was the specific component of $\mathrm{S} 1$ stimulated in our study, is located in the posterior bank of the central sulcus and receives its major activating inputs from the ventroposterior nucleus of the thalamus ${ }^{[28]}$, including C-fibers responsive to histamine ${ }^{[29]}$. Thus, one possible explanation of our finding is that cTBS of contralateral S1 leads to a reduced excitability to input from histamineresponsive $\mathrm{C}$-fibers, resulting in reduced itch intensity. An alternative (though not mutually exclusive) explanation is that disrupting activity in S1 results in a top-down inhibition of itch including the periaqueductal grey (PAG) ${ }^{[1,30]}$. The PAG, which serves as a functional and anatomical interface between forebrain and lower brain stem ${ }^{[31]}$, is anatomically well-connected with the main nodes of the cortical itch network, including insula, anterior cingulate cortex and S1 ${ }^{[32]}$. Disrupting activity in contralateral S1 may trigger a descending inhibition via the PAG. Such an interpretation fits well with neurostimulation studies from the related domain of pain, where evidence suggests that reducing excitability of contralateral $S 1$ interferes with nociceptive processing ${ }^{[33-36]}$. For example, Torta et al. ${ }^{[33]}$ observed that cTBS of contralateral, but not ipsilateral, S1 reduces the perceived intensity of nociceptive stimuli. Since cTBS did not disrupt processing of tactile stimuli in that study, the authors speculate that the analgesic effect of contralateral cTBS is mainly the result of a modulation of descending pathways controlling the spinal transmission of ascending nociceptive inputs.

This article is protected by copyright. All rights reserved. 
A possible explanation of why the itch reduction following S1 stimulation only reached full statistical significance when mean itch was used as the outcome measure, but not when peak itch was used, may be due to the reduced reliability of the peak itch measure. Since the peak itch measure reflects a single rating of a participant, it is more influenced by unsystematic random influences than the mean of an itch rating timecourse, where the averaging process tends to reduce the influence of unsystematic variation.

Although the secondary somatosensory cortex (S2) was found to be reliably activated across the reviewed neuroimaging studies (see supplementary online material), the present study found no evidence that disrupting activity in S2 via cTBS results in a reduction of itch intensity. This could indicate a functional division between somatosensory cortices in itch, with a sensory-discriminative role for S1 and a more general role for S2 of recognizing and attending to pruritic stimuli. In the domain of pain, both animal and human studies have demonstrated that S1 faithfully encodes perception of stimulus intensity ${ }^{[37]}$, suggesting a sensory-discriminative functional role of this area. In contrast, S2 shows a sharp increase in amplitude only when the stimulus intensity is well above the pain threshold and typically does not show a clear dose-response relationship for stimulation in the noxious range ${ }^{[37-40]}$. On the basis of such findings, it has been argued that $\mathrm{S} 2$ may have a specific role in the recognition of and attention toward painful stimuli, rather than intensity processing ${ }^{[37]}$. The findings of the present study (cTBS of S1, but not of S2, reduces itch intensity) as well the study by Drzezga et al. ${ }^{[17]}$, who observed a correlation between pruritic dose and S1, but not S2 activity, are in line with the suggestion made by Mochizuki and colleagues ${ }^{[1]}$ that a similar functional division between S1 and S2 may exist for itch as well. Future brain stimulation studies using different outcome measures, such spontaneous scratching frequency, could be very illuminating in this respect.

The current study also found no significant reduction of itch intensity following IFG stimulation. Although this area is reliably activated in acute itch, the functional role of the IFG in itch is currently not well understood. Our results suggest that this part of the prefrontal cortex does not play a critical role in the perception of acute itch intensity. However, this does not rule out the possibility that this area is crucial for other aspects of itch processing, such as stimulus localization ${ }^{[10]}$ or motor planning of the scratch response ${ }^{[8,9]}$.

It should be noted that the lack of cTBS effects in areas S2 and IFG could also be related to stimulation intensity. In the current study, we used a fixed stimulation intensity for all subjects and all brain areas. The average cortical depth of the three stimulation sites, as estimated on the MNI brain template, were $26.6 \mathrm{~mm}$ (S1), $31 \mathrm{~mm}$ (IFG) and $36 \mathrm{~mm}$ (S2). Since the strength of the induced magnetic field decreases exponentially as the distance from the scalp increases, this raises the possibility that stimulation intensity may not have been sufficient to perturb activity in more deeply located cortical areas, in particular S2. Future studies could improve upon this by determining individual intensity thresholds for each subject and area, but for non-motor areas this requires statistical modelling of the induced electric fields; a procedure which is currently being developed ${ }^{[41]}$.

This article is protected by copyright. All rights reserved. 
A final aspect to consider is the statistical sensitivity (i.e., power) of our study to detect an antipruritic effect in S2 and IFG, should one exist in the population. Assuming that such a putative effect of S2 and IFG stimulation exists and is of similar size as the one we report here for S1 stimulation, the present study with a sample size of $\mathrm{N}=19$ had a power of .71 to detect such an effect. Since the recommend level of power is at least $.8^{[42]}$, the present study was thus slightly underpowered with respect to S2 and IFG and we note that we may have missed a potential antipruritic effect for those areas.

In summary, the present study provided evidence for an anti-pruritic effect of inhibitory stimulation of contralateral S1. This observation might be important in future studies on brain interventions for the treatment of itch. More work is needed to determine the degree to which cTBS of S1 disrupts other sensory domains beyond itch, such as thermal and mechanical sensations. Finally, it would be important to test whether the anti-pruritic effect transfers to histamine-independent pathways (e.g., using cowhage as pruritogen) and clinical populations.

\section{Acknowledgements}

This work was supported by the Department of Psychology, as well as a grant from the British Skin Foundation (project number: 7011s) awarded to $\mathrm{HH}$.

\section{Conflict of Interest Statement}

We have no conflicts of interest to declare.

\section{Author Contributions Statement}

$\mathrm{HH}$ and IS designed the research. OJ performed the research. $\mathrm{OJ}$ and $\mathrm{HH}$ analysed the data. $\mathrm{HH}, \mathrm{OJ}$ and IS wrote the paper. All authors have read and approved the final manuscript.

\section{References}

[1] H. Mochizuki, R. Kakigi. Central mechanisms of itch. Clin Neurophysiol. 2015;126(9):16501660 .

[2] A. Ikoma, M. Steinhoff, S. Stander, G. Yosipovitch, M. Schmelz. The neurobiology of itch. Nat Rev Neurosci. 2006;7(7):535-547.

[3] H. Knotkova, R.K. Portenoy, R.A. Cruciani. Transcranial direct current stimulation (tDCS) relieved itching in a patient with chronic neuropathic pain. Clin J Pain. 2013;29(7):621-622.

[4] K. Nakagawa, H. Mochizuki, S. Koyama, S. Tanaka, N. Sadato, R. Kakigi. A transcranial direct current stimulation over the sensorimotor cortex modulates the itch sensation induced by histamine. Clin Neurophysiol. 2016;127(1):827-832.

[5] Y.Z. Huang, M.J. Edwards, E. Rounis, K.P. Bhatia, J.C. Rothwell. Theta burst stimulation of the human motor cortex. Neuron. 2005;45(2):201-206.

[6] P.L. Lockwood, G.D. Iannetti, P. Haggard. Transcranial magnetic stimulation over human

This article is protected by copyright. All rights reserved. 
secondary somatosensory cortex disrupts perception of pain intensity. Cortex. 2013;49(8):2201-2209.

[7] T. Valmunen, A. Pertovaara, T. Taiminen, A. Virtanen, R. Parkkola, S.K. Jaaskelainen. Modulation of facial sensitivity by navigated rTMS in healthy subjects. Pain. 2009;142(12):149-158.

[8] U. Darsow, A. Drzezga, M. Frisch, et al. Processing of histamine-induced itch in the human cerebral cortex: a correlation analysis with dermal reactions. J Invest Dermatol. 2000;115(6):1029-1033.

[9] J.C. Hsieh, O. Hagermark, M. Stahle-Backdahl, et al. Urge to scratch represented in the human cerebral cortex during itch. J Neurophysiol. 1994;72(6):3004-3008.

[10] Y. Ishiuji, R.C. Coghill, T.S. Patel, Y. Oshiro, R.A. Kraft, G. Yosipovitch. Distinct patterns of brain activity evoked by histamine-induced itch reveal an association with itch intensity and disease severity in atopic dermatitis. Br J Dermatol. 2009;161(5):1072-1080.

[11] R.C. Oldfield. The assessment and analysis of handedness: The Edinburgh inventory. Neuropsychologia. 1971;9(1):97-113.

[12] E.J. Williams. Experimental designs balanced for the estimation of residual effects of treatments. Australian Journal of Scientific Research, Series A: Physical Sciences. 1949;2:149-168.

[13] Z.D. Deng, S.H. Lisanby, A.V. Peterchev. Electric field depth-focality tradeoff in transcranial magnetic stimulation: simulation comparison of 50 coil designs. Brain Stimul. 2013;6(1):113.

[14] T. Nyffeler, D. Cazzoli, C.W. Hess, R.M. Muri. One session of repeated parietal theta burst stimulation trains induces long-lasting improvement of visual neglect. Stroke. 2009;40(8):2791-2796.

[15] T. Nyffeler, D. Cazzoli, P. Wurtz, et al. Neglect-like visual exploration behaviour after theta burst transcranial magnetic stimulation of the right posterior parietal cortex. Eur J Neurosci. 2008;27(7):1809-1813.

[16] E.M. Wassermann. Variation in the response to transcranial magnetic brain stimulation in the general population. Clin Neurophysiol. 2002;113(7):1165-1171.

[17] A. Drzezga, U. Darsow, R.D. Treede, et al. Central activation by histamine-induced itch: analogies to pain processing: a correlational analysis of $\mathrm{O}-15 \mathrm{H} 2 \mathrm{O}$ positron emission tomography studies. Pain. 2001;92(1-2):295-305.

[18] D.M. Lloyd, E. Hall, S. Hall, F.P. McGlone. Can itch-related visual stimuli alone provoke a scratch response in healthy individuals? Br J Dermatol. 2013;168(1):106-111.

[19] R.H. LaMotte, S.G. Shimada, B.G. Green, D. Zelterman. Pruritic and nociceptive sensations and dysesthesias from a spicule of cowhage. J Neurophysiol. 2009;101(3):1430-1443.

[20] O. Jones, I.C. Schindler, H. Holle. Assessing Acute Itch Intensity: General Labelled Magnitude Scale is More Reliable than Classic Visual Analogue Scale. Acta Derm Venereol. 2017;97(3):375-376.

[21] N.Q. Phan, C. Blome, F. Fritz, et al. Assessment of pruritus intensity: prospective study on validity and reliability of the visual analogue scale, numerical rating scale and verbal rating scale in 471 patients with chronic pruritus. Acta Derm Venereol. 2012;92(5):502-507.

[22] G. Heyer, O.P. Hornstein, H.O. Handwerker. Skin reactions and itch sensation induced by epicutaneous histamine application in atopic dermatitis and controls. J Invest Dermatol. 1989;93(4):492-496.

[23] M.-L. Essink-Bot, M.C. Stuifbergen, W.-J. Meerding, C.W.N. Looman, G.J. Bonsel, V.g. the. Individual differences in the use of the response scale determine valuations of hypothetical health states: an empirical study. BMC Health Services Research. 2007;7:62-62.

[24] B.G. Tabachnik, L.S. Fidell. Using multivariate statistics. 2 ed. New York: HarperCollins; 1989.

[25] L. Herde, C. Forster, M. Strupf, H.O. Handwerker. Itch induced by a novel method leads to limbic deactivations a functional MRI study. J Neurophysiol. 2007;98(4):2347-2356.

[26] A.D. Papoiu, R.C. Coghill, R.A. Kraft, H. Wang, G. Yosipovitch. A tale of two itches. Common features and notable differences in brain activation evoked by cowhage and histamine induced itch. Neuroimage. 2012;59(4):3611-3623.

This article is protected by copyright. All rights reserved. 
[27] M. Valet, F. Pfab, T. Sprenger, et al. Cerebral processing of histamine-induced itch using short-term alternating temperature modulation--an FMRI study. J Invest Dermatol. 2008;128(2):426-433.

[28] C. Keysers, J.H. Kaas, V. Gazzola. Somatosensation in social perception. Nat Rev Neurosci. 2010;11(6):417-428.

[29] R.H. LaMotte, X. Dong, M. Ringkamp. Sensory neurons and circuits mediating itch. Nat Rev Neurosci. 2014;15(1):19-31.

[30] E. Carstens. Responses of rat spinal dorsal horn neurons to intracutaneous microinjection of histamine, capsaicin, and other irritants. J Neurophysiol. 1997;77(5):2499-2514.

[31] E.E. Benarroch. Periaqueductal gray: an interface for behavioral control. Neurology. 2012;78(3):210-217.

[32] E. Sillery, R.G. Bittar, M.D. Robson, et al. Connectivity of the human periventricularperiaqueductal gray region. J Neurosurg. 2005;103(6):1030-1034.

[33] D.M. Torta, V. Legrain, M. Algoet, E. Olivier, J. Duque, A. Mouraux. Theta burst stimulation applied over primary motor and somatosensory cortices produces analgesia unrelated to the changes in nociceptive event-related potentials. PLoS One. 2013;8(8):e73263.

[34] C. Poreisz, A. Antal, K. Boros, N. Brepohl, G. Csifcsak, W. Paulus. Attenuation of N2 amplitude of laser-evoked potentials by theta burst stimulation of primary somatosensory cortex. Exp Brain Res. 2008;185(4):611-621.

[35] A. Antal, N. Brepohl, C. Poreisz, K. Boros, G. Csifcsak, W. Paulus. Transcranial direct current stimulation over somatosensory cortex decreases experimentally induced acute pain perception. Clin J Pain. 2008;24(1):56-63.

[36] C.A. Porro, M. Martinig, P. Facchin, M. Maieron, A.K. Jones, L. Fadiga. Parietal cortex involvement in the localization of tactile and noxious mechanical stimuli: a transcranial magnetic stimulation study. Behav Brain Res. 2007;178(2):183-189.

[37] L. Timmermann, M. Ploner, K. Haucke, F. Schmitz, R. Baltissen, A. Schnitzler. Differential coding of pain intensity in the human primary and secondary somatosensory cortex. $J$ Neurophysiol. 2001;86(3):1499-1503.

[38] K. Bornhovd, M. Quante, V. Glauche, B. Bromm, C. Weiller, C. Buchel. Painful stimuli evoke different stimulus-response functions in the amygdala, prefrontal, insula and somatosensory cortex: a single-trial fMRI study. Brain. 2002;125(Pt 6):1326-1336.

[39] W.K. Dong, E.H. Chudler, K. Sugiyama, V.J. Roberts, T. Hayashi. Somatosensory, multisensory, and task-related neurons in cortical area $7 \mathrm{~b}(\mathrm{PF})$ of unanesthetized monkeys. $J$ Neurophysiol. 1994;72(2):542-564.

[40] M. Frot, M. Magnin, F. Mauguiere, L. Garcia-Larrea. Human SII and posterior insula differently encode thermal laser stimuli. Cereb Cortex. 2007;17(3):610-620.

[41] A. Thielscher, A. Antunes, G.B. Saturnino. Field modeling for transcranial magnetic stimulation: A useful tool to understand the physiological effects of TMS? Conf Proc IEEE Eng Med Biol Soc. 2015;2015:222-225.

[42] J. Cohen. A power primer. Psychol Bull. 1992;112(1):155-159.

[43] S.B. Eickhoff, T. Paus, S. Caspers, et al. Assignment of functional activations to probabilistic cytoarchitectonic areas revisited. Neuroimage. 2007;36(3):511-521.

\section{Figure Captions}

Figure 1 Overview of target regions, including MNI coordinates and most probably anatomic region in the Anatomy Toolbox 2.2c ${ }^{[43]}$. Right IFG (51, 10, 7); left S1 (-32, -35, 62); left S2 (-47, -21, 13); SPL (-3, -62, 71). Abbreviations: IFG: Inferior Frontal Gyrus; SI: Primary Somatosensory Cortex; SII: Secondary Somatosensory Cortex; OP: Operculum Parietale; SPL: Superior Parietal Lobe.

This article is protected by copyright. All rights reserved. 
Figure 2 Itch intensity results for each stimulation condition. a) Mean time series ( $N=19)$ of normalized itch ratings (total duration: 600 s). b) Peak itch for each condition, averaged across participants. c) Mean itch for each condition, averaged across participants. Error bars indicate the standard error of the mean.
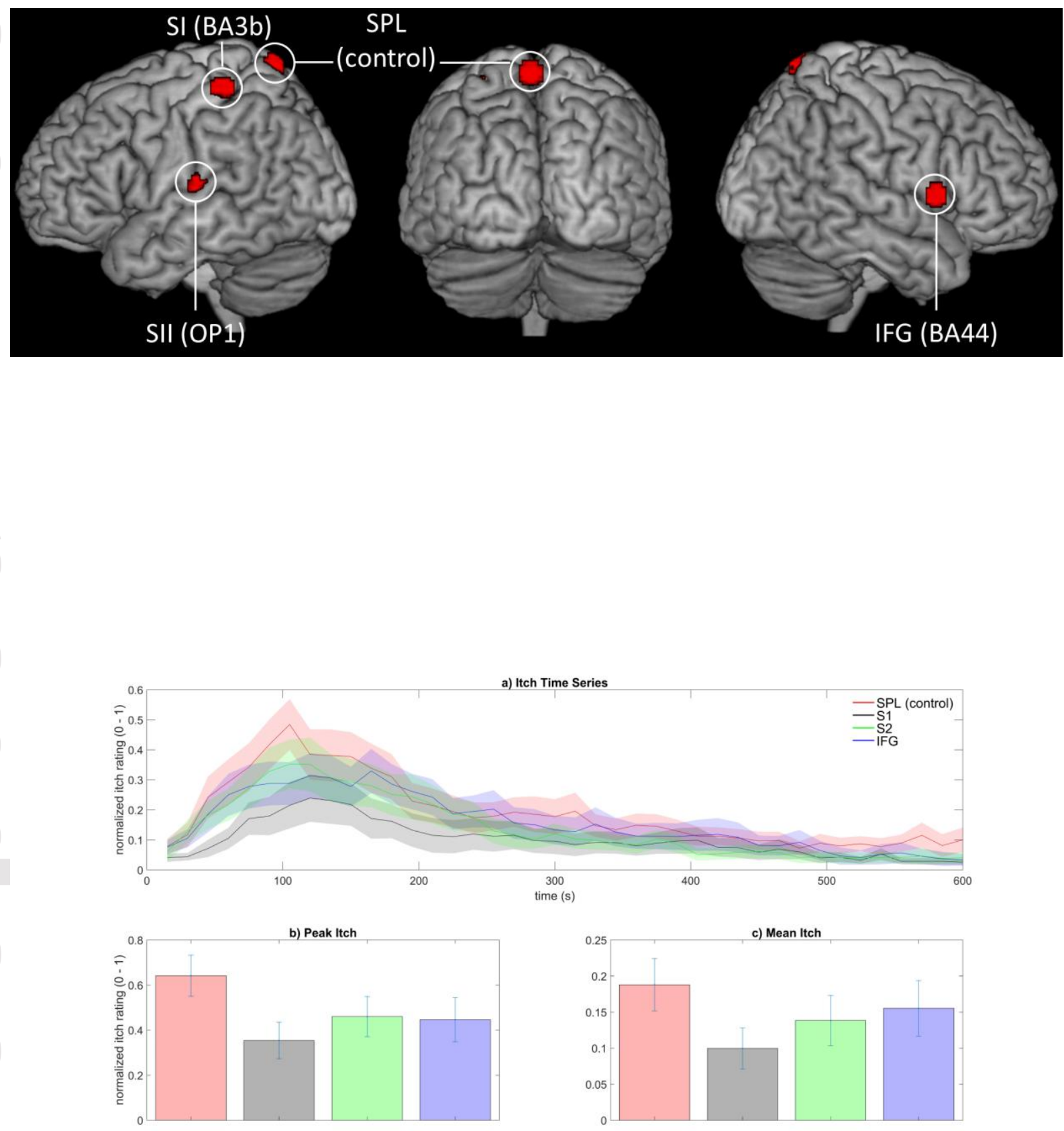

This article is protected by copyright. All rights reserved. 